\title{
Nanoparticles Functionalized with Ligands of Cell Surface Nucleolin for Cancer Therapy and Diagnosis
}

\author{
Maha Sader ${ }^{1,2}$, José Courty ${ }^{1,2 *}$ and Damien Destouches ${ }^{1,2}$
}

${ }^{1}$ Université Paris-Est, UPEC, F-94000, Créteil, France

${ }^{2}$ CNRS, ERL 9215, Laboratoire de Recherchesur la Croissance Cellulaire, la Réparation et la Régénération Tissulaires (CRRET), F-94000 Créteil, France

\begin{abstract}
Conventional cancer chemotherapies are often limited by their non-targeted nature and their inadequate delivery to the tumor affecting the normal tissues and leading to toxic adverse effects. In order to improve the anticancer efficacy and safety of these drugs, as well as the diagnosis capacity of imaging agents, nanoparticles drug delivery system has been developed combining ligands enabling tumor targeting at a cellular level and drug carrier capacity. Nucleolin $(\mathrm{NCL})$ is a multifunctional protein that could shuttle from nucleolus to nucleoplasm, cytoplasm and cell surface. This ribonucleo protein over expressed at the cell surface of cancer cells is involved in many cancer processes supporting tumorigenesis such as cell proliferation and apoptosis. Additionally, NCL expression is enhanced in angiogenic vessels, enabling multi-targeting strategies toward the tumor microenvironment. In this context, several compounds targeting $\mathrm{NCL}$, such as the aptamer AS1411, the peptide F3 and the multivalent pseudopeptide N6L, have been developed and investigated for cancer therapy. Due to their cancer cell targeting capacities, these compounds have been evaluated to mediate highly specific and effective nanoparticles for drug delivery to the tumor. In this report, we present a review of literature focusing on drug-loaded nanoparticles conjugated with these nucleolin ligands, strikingly emphasizing the success of such a strategy.
\end{abstract}

Keywords: Cancer; Cell surface nucleolin; Nanoparticles; As1411; F3; N61; Tumor targeting; Cancer therapy; Cancer diagnosis; Cancer theragnostic

Abbreviations: NP: Nanoparticle; N6L: NucAnt 6L; DOX: Doxorubicin; siRNA: Small interfering RNA; MRI: Magnetic Resonance Imaging; PEG-PLGA: Poly Ethylene glycol-Functionalized Poly (D,LLactic-Co-Glycolic Acid); VEGF: Vascular Endothelial Growth Factor; PCR: Polymerase Chain Reaction; GFP: Green Fluorescent Protein

\section{Introduction}

Cancer remains a significant health problem worldwide accounting in 2012 for 14.1 million of new cases and 8.2 million deaths [1]. The therapeutic arsenal for cancer is composed of surgery and radiotherapy used for local and non-metastatic cancers and anticancer drugs (chemotherapy, hormono-therapy and targeted therapies) more often used in metastatic cancers. Chemotherapeutic drugs mechanism is based on inhibition of cell division of cancer cells but, unfortunately these compounds act also on several normal cells needing high renewal such as bone marrow, gastrointestinal or hair follicle cells. In fact, clinical efficacy of chemotherapeutic agents is often hindered by serious adverse effects, caused by an inadequate delivery to the tumor due to unfavorable pharmacokinetic profile and to a non-specific-tumor-cellkilling. For instance, the clinical efficacy of paclitaxel, a taxane molecule inhibiting microtubule depolymerization, is often limited by its poor aqueous solubility, and hypersensitivity reactions and neurotoxicity induced by its solvent cremophor/ethanol [2]. Doxorubicin (DOX), one of the most widely used antineoplastic agents inhibiting the topoisomerase II and intercalating in DNA and RNA, is well known for its cardiotoxic side effects [3]. In this context, one major challenge in cancer research remain to improve treatments efficiency along with patient quality of life by reducing side effects of these most frequently used therapeutic arms. Other great needs in cancer therapy are to improve early disease detection in order to prevent cancer progression to advanced stages and metastasis, to determine the characteristics (location, stage and grade) of the tumor to adapt the treatment so as to achieve the best outcome for each patient, and to allow real-time monitoring of the treatment. Many images techniques providing both anatomical and functional information have shown great promises in cancer diagnosis, such as radionuclide-based positron emission tomography (PET), magnetic resonance imaging (MRI) and fluorescence-based optical imaging. However, single imaging modality and unfavorable pharmacokinetic profile of diagnostic agents still constitute the major limits [4].

In order to overcome these therapeutic and diagnostic limits, two strategies have been widely investigated: the nanoparticle (NP) drug delivery system and targeted therapies that also could be combined as a theranostic tool.

The first nanotechnology tool consists in loading chemotherapeutic drugs into a NP subtype. NPs are defined as objects having at least one dimension that ranges between 1 and 100 nanometers. NPs are potentially able to interact with biological entities having similar size, such as proteins or tumor cells [5]. There are several types of NPs that can be classified according to their composition into organic and inorganic NPs. Organic NPs include liposomes, micelles, solid lipid NPs, dendrimers and polymeric NPs (poly(ethylene glycol) (PEG)functionalized poly (D,L-lactic-co-glycolic acid) (PLGA) (PEGPLGA), hydrogels NPs, polyacrylamide). The most studied inorganic NPs are gold NPs, quantum dots, silica, carbon and magnetic NPs [6]. NPs have been widely investigated to transport to the tumor, many active compounds that have poor pharmacokinetic properties such as

*Corresponding author: José Courty, Laboratoire CRRET, ERL CNRS 9215, Université Paris-Est, 61 Avenue du général de Gaulle, Créteil, F-94000 France, Tel: 33-1-45-17-17-99; Fax: 33-1-45-17-18-16; E-mail: courty@u-pec.fr

Received June 15, 2015; Accepted July 02, 2015; Published July 12, 2015

Citation: Sader M, Courty J, Destouches D (2015) Nanoparticles Functionalized with Ligands of Cell Surface Nucleolin for Cancer Therapy and Diagnosis. J Nanomed Nanotechnol 6: 310. doi:10.4172/2157-7439.1000310

Copyright: $\odot 2015$ Sader M, et al. This is an open-access article distributed unde the terms of the Creative Commons Attribution License, which permits unrestricted use, distribution, and reproduction in any medium, provided the original author and source are credited. 
rapid degradation, elimination or poor absorption [6]. In addition, NPs constitute a platform for multifunctional multi-drug loading, enabling to improve efficacy in cancer therapy, to overcome the limitation of a single imaging modality in cancer diagnosis, and to combine therapy and diagnosis within a single formula, a strategy known as theranostic [7]. As for the concept of targeted therapies that rose in the early 1990s, it is based on targeting specific molecular abnormalities in cancer cells, such as a receptor on the cell surface, an intracellular protein or gene. Thus, combining both strategies by conjugating drug transporting NPs with tumor targeting molecules favors drug delivery's selectivity, efficacy and therapeutic index $[8,9]$. It is in this context that the current review is being addressed.

Among widely investigated receptors for cancer targeting, nucleolin (NCL) seems to be a promising molecule. This ribonucleo protein is involved in several cellular processes such as ribosomal assembly and maturation and also in tumorigenesis. NCL is over-expressed on the cell surface of many cancer cells and accumulating evidences validated cell surface nucleolin as a very promising target in the treatment of cancer. These studies have been commented in recent reviews that summarize publications and patents related to the use of NCL ligands in cancer therapies [10-12].

NPs functionalized with NCL targeting ligands which exhibit high affinity toward tumor cells, have been an approach aimed at improving anti-cancer efficacy and safety of chemotherapeutic agent as well as imaging for cancer diagnosis. To date, known NCL ligands that are already used to functionalize NP are: the aptamer AS1411 [13], the F3 peptide [14] and the multivalent pseudopeptide N6L [15]. NPs functionalized with these NCL ligands as well as the roles of these NPs in cancer constitute the focus of this review.

\section{NCL: A promising target for cancer therapy}

NCL, initially called C23, is a protein composed of 707 amino acids protein with an apparent molecular mass of $100-110 \mathrm{kDa}$, and ubiquitously expressed in most eukaryotic cells in which it represents an important multi-functional protein. NCL is mainly localized in the nucleolus of quiescent cells where it makes $10 \%$ of total proteins $[16,17]$. It is also found in the nucleus [18], the cytoplasm [19] and on cell surface [20]. NCL displays different functions depending on its localization. In the nucleolus, NCL was essentially described for its role in ribosome synthesis where it contributes to rDNA transcription, pre-RNA maturation and the assembly of ribosomal subunits [17]. In the nucleus, NCL is involved in DNA replication and reparation and is implicated in mRNA post-transcriptional regulation in the cytoplasm [17]. At cell surface, NCL binds to several ligands involved in cell proliferation, apoptosis, angiogenesis and microorganism/cell binding [12].

NCL held the attention as a target for cancer therapies accounting to its implication in tumorigenesis but also and mainly for its increase expression in cancer cells. In fact, the nucleolar activity as ribosome synthesis is increased in rapidly dividing cells compared to quiescent cells. The level of NCL synthesis is correlated with the rate of cell doubling, its expression is thus greater in many tumor cells types $[21,22]$. NCL is a marker of several human cancer such as colorectal gastric, lung, cervical and breast carcinomas, melanoma and glioblastoma [23-34]

In each compartment, the rise of NCL expression participates to tumorigenesis. In the nucleolus, the high level of NCL increases RNA polymerase I transcription contributing to hyperactivation of rDNA transcription and thus to a high level of protein synthesis required for a high proliferation rate [35]. In the nucleoplasm, NCL regulates the transcription of genes involved in cancer such as Interleukin-9 receptor which displays proliferative and anti-apoptotic activities [36] and the vascular endothelial growth factor (VEGF), receptor of one of the main factor involved in angiogenesis during tumorigenesis and metastasis [37]. NCL also regulates the expression of a specific subset of miRNA miR-21, miR-221, miR-222, and miR-103 involved in breast cancer initiation, progression and drug resistance [30]. In the cytoplasm, NCL binds and regulates mRNA involved in apoptosis and tumorigenesis. NCL binds to the anti-apoptotic proteins $\mathrm{Bcl}-2$ and Bcl-xl mRNA allowing tumor cells to run off from apoptosis $[38,39]$. Interacting with p53 mRNA, it prevents its translation and thus protects cells from apoptosis $[40,41]$. Furthermore, NCL enhances translation of many mRNA encoding for oncogenic proteins [42]. Cell surface NCL has been reported to act as a receptor for several growth factor such as midkine [43] and pleiotrophin [43-45] involved in angiogenesis and tumor growth, hepatocyte growth factor (HGF) involved in the mechanisms of metastasis and cell adhesion [46] and P-selectin that induces signaling pathways of cell adhesion and invasion [47]. In addition, the implication of cell surface NCL in proliferative diseases may be related to the fact that it exists in a protein complex of $500-\mathrm{kDa}$, including several proteins involved in cancer progression. As described by Krust et al., this complex is composed at least of eight proteins in leukemia CEM cells: Wnt-A and Wnt-B involved in the differentiation of cancer cells and normal cells; the antigen Ku80 expresses on the surface of various tumor cell lines such as leukemia's and solid tumors; the SRP68 and SRP72 subunits of a ribonucleo protein complex involved in the synthesis and translocation of secretory proteins to the membrane; the $\mathrm{C} 1 \mathrm{q}$ complement receptor $\mathrm{gC} 1 \mathrm{q}-\mathrm{R}$ involved in tumor progression, and finally the S4 and S6 ribosomal proteins that could, like several other ribosomal proteins, play a role in cancer [48]. The overexpression of cell surface NCL was reported in many cancer cell lines, such as human breast carcinoma cell lines (MDA-MB-231, MDA-MB-435), human carcinoma (LNCaP, HeLa, G401), leukemia (Jurkat, HuT 78, CEM) $[15,49]$ and in endothelial cells stimulated by VEGF [50]. Thus, using NCL ligands enables selective targeting of two distinct cell populations within the tumor microenvironment: cancer cells and endothelial cells constituting the angiogenic blood vessels. As angiogenesis promotes cell survival and tumor metastasis [51], this dual targeting yields many advantages: endothelial cells are less able to acquire drug resistance and are more accessible to the drug injected in the vascular compartment injection, avoiding low drug accumulation into the tumor owing to high interstitial pressure $[52,53]$. Thus, a formulation enabling the concentration of the therapeutic agent into endothelial and tumor cells is believed to ultimately improve cancer treatment efficiency.

Moreover, as NCL participates in binding and endocytosis/ macropinocytosis processes $[54,55]$ and shuttles between the cell surface, the cytoplasm and the nucleus [56]. Drug loaded by NPs could be transported through NCL to cell nucleus which could improve the drug delivery and cause greater cell growth inhibition. Finally, as NCL is always overexpressed in almost all cancer cells, strategies targeting NCL will not be limited to a specific category of patient as some current strategies depends on the expression or not of the targeted receptor.

Considering all these advantages of NCL targeting in cancer therapies, AS1411, F3 and N6L compounds were developed and studied for their targeting properties and/or antitumor activities. The three molecules have been conjugated to NPs loaded with drugs, genes or imaging agents and investigated by in vitro and in vivo studies for cancer therapy and diagnosis. A number of these studies highlighting 
the success of this strategy are reviewed in this work and summarized in Table 1.

\section{AS1411 aptamer conjugated NPs targeting NCL}

Aptamers are synthetic single-stranded DNA or RNA oligonucleotides that bind to target molecules with high affinity and specificity enabled through their 3-dimensional structure [57]. As ligands for molecular targeting, aptamers exhibit significant advantages compared to antibodies in terms of low cost production, easier scaling up, avoiding animal use and convenience to be transported at ambient temperature after synthesis. Additionally, aptamers are smaller in size $(\sim 1-2 \mathrm{~nm},<10 \mathrm{kDa})$ compared to antibodies $(\sim 10 \mathrm{~nm}, \sim 155 \mathrm{kDa})$ allowing better tissue and tumor penetration [58].

AS1411, a guanosine-riche DNA aptamer (26-mer, $7.8 \mathrm{kDa}$ ), is currently in phase II clinical trials [57,59]. It binds to NCL with affinity ranging from $\mathrm{pM}$ to low $\mathrm{nM}$ [13]. Indeed, plasma membrane NCL has been reported as a receptor for AS1411 in many tumor cells such as MV4-11 leukemia cells [33], MDA-MB-231 and HeLa cells [60]. By blocking several functions of NCL, AS1411 exhibits antitumor activities. It destabilizes Bcl-2 [61], blocks the anti-apoptotic pathway of NF-kB [62] and reduces NCL-dependent miRNA levels [30]. It results in in vitro cell growth inhibition in various human tumor cell lines including MCF-7 [61,62], HeLa, DU-145 and A549 [62], and in breast cancer tumor growth inhibition associated to reduced metastasis in in vivo MDA-MB-231 xenograft models [30]. More recently, the team of Bates has demonstrated that the antiproliferative activity of AS1411 correlated with its capacity to stimulate macro pinoicytosis through activation of Rac1 [63] causing methuosis [64], a new type of non-apoptotic cell death. Thus, AS1411 exhibits dual properties of targeting and killing tumor cells. AS1411 was also used as a potential tumor-targeting agent for imaging in various cancer models such as prostate [65], glioma [66], lung [67], cervical [68].

Hence, several NPs formulations conjugated with AS1411 have been successfully investigated in therapy and diagnosis of various cancer types. These NPs showed targeting specificity, enhanced tumor cytotoxicity, a reduced toxicity of the chemotherapeutic agent loaded as well as an enhanced imaging signal.

Many of these formulations have been investigated in glioma tumors, taking advantages in NCL over expression in endothelial angiogenesis vessel cells that enables specific accumulation of the drug loaded in the glioma compared to strategies overcoming BBB (blood-brain barrier) that could favor non-specific distribution in the central nervous system. Using C6 rat glioma cells, Guo et al. reported an enhanced anti-proliferative effect of PTX loaded PEG-PLGA NPs when linked with AS1411, certainly owed to high cellular association facilitated by the NCL-AS1411 interaction. Pharmacokinetic studies performed on rats that received intravenous injections showed significant longer circulation time of both targeted and non-targeted NPs formulations than $\mathrm{Taxol}^{\circ}$ due to the enhanced stability of the PEG-PLGA formulation. Anti-glioma efficacy evaluated in C6 glioma xenografts bearing nude mice after intravenous administration, showed that PTX accumulation in the tumor was significantly improved by AS1411 conjugation to NPs, which resulted in tumor inhibition and a significantly prolonged animal survival evaluated on Wistar rats bearing C6 glioma [69]. Other formulations grafted with AS1411 were a worthy object of studies for simultaneous treatment and diagnosis of glioma. Kim et al. have developed an AS1411 grafted theragnostic probe of magnetic fluorescent NPs loaded with miRNA-221, a molecular probe that contains perfectly complimentary oligonucleotides against

\begin{tabular}{|c|c|c|c|c|}
\hline NCL Ligand & NPs & Drug/gene/dye delivered & Application & Ref \\
\hline \multirow{8}{*}{$\begin{array}{l}\text { AS1411 } \\
\text { aptamer }\end{array}$} & PEG-PLGA & PTX & Cancer therapy Rat glioma (C6 cells) and ectopic xenorafts & [42] \\
\hline & Magnetic fluorescent & $\begin{array}{l}\mathrm{m} \text { iR N A-221 molecular } \\
\text { beacon probe }\end{array}$ & Cancer theragnostics Rat glioma (C6 cells) and ectopic xenografts & [43] \\
\hline & Magnetic fluorescent & ${ }^{67}$ Ga-citrate & Cancer imaging Rat glioma ( $\mathrm{C} 6$ cells) and ectopics xenografts & [44] \\
\hline & Liposomes & SiBRAF & Cancer therapy Human melanoma (A375 cells) and ectopic & [45] \\
\hline & Albumin & PTX & Cancer therapy Human Breast cancer (MCF -7 cells) & [46] \\
\hline & Liposomes & DOX & Cancer therapy Human Breast cancer (MCF -7 cells) and ectopic xenografts & [47] \\
\hline & Gold nanostars & & $\begin{array}{l}\text { Cancer therapy Human carcinoma (A-549, A498, MD A-MB-231, HeLa), sarcoma } \\
\text { (HT-1080), melanoma (SK-MEL-2), glioblastoma (U-87MG) }\end{array}$ & [48] \\
\hline & Gold nanostars & & $\begin{array}{l}\text { Observation of NPs cancer cell nucleus interactions Human cervical cancer (HeLa } \\
\text { cells) }\end{array}$ & [49] \\
\hline \multirow[t]{7}{*}{ F3 peptide } & Hydrogel & DOX & Cancer therapy Rat glioma (9L cells) & {$[50]$} \\
\hline & PEG-PLGA & PTX & Cancer therapy Rat glioma (C6 cells) and orthotopic xenograft & [51] \\
\hline & Polyacrylamide & $\begin{array}{l}\text { Methylene blue coomassie } \\
\text { blue indocyanine green }\end{array}$ & Cancer imaging Rat glioma (9L cells) & [52] \\
\hline & Liposomes & DOX & $\begin{array}{l}\text { Cancer therapy Human breast cancer cells (MDA-MB-231, MCF-7) Human } \\
\text { melanoma (MDA-MB-435S cells) }\end{array}$ & [53] \\
\hline & Liposomes & DOX+C6-ceramide & $\begin{array}{l}\text { Cancer therapy Human breast cancer cells (MDA-MB-231 cells) Human melanoma } \\
\text { (MDA-MB-435S cells) }\end{array}$ & [54] \\
\hline & Carbon nanotubes & & $\begin{array}{l}\text { Cancer near infrared light therapy Human breast cancer (MCF-7 cells) Human aortic } \\
\text { endothelial cells (HAAE-1) }\end{array}$ & {$[55]$} \\
\hline & Liposomes & $\begin{array}{l}\text { siRNA GFP (Green } \\
\text { Fluorescent Protein) }\end{array}$ & $\begin{array}{l}\text { Cancer therapy Human breast cancer (MDA-MB-231 and MD A MB-435S cells) } \\
\text { Human microvascular endothelial cell HMEC-1 }\end{array}$ & [56] \\
\hline \multirow{4}{*}{$\begin{array}{l}\text { N6L pseudo } \\
\text { peptide }\end{array}$} & Iron oxide magnetic NPs & & Cancer therapy Human breast cancer (MDA-MB-231cells) and ectopic xenografts & MS15 \\
\hline & Iron oxide magnetic NPs & DOXX & Cancer therapy Human breast cancer (MDA-MB-231cells) and ectopic xenografts & {$[57]$} \\
\hline & DNA & GFP/Luciferase reporter gene & Cancer therapy Human cervical cancer HeLa cells & [58] \\
\hline & DNA & GFP/Luciferase reporter gene & Study of NCL-DNA NPs complex fate Human cervical cancer HeLa cells & [59] \\
\hline
\end{tabular}

The table summarizes the different formulations of NPs functionalized with the anti-NCL ligands AS1411, F3 and N6L as well as non-functionalized NPs targeting NCL, with the different in vitro and in vivo models used for cancer therapy and diagnosis NPs studies.

Table 1: NPs targeting NCL for cancer therapy and diagnosis. 
the target miRNA. Hence, these NPs simultaneously target cancer tissue and treat the intracellular expressed miRNA-221 involved in carcinogenesis and highly expressed in many cancer cells including C6 glioma cells [70]. AS1411 promoted NPs plasma membrane targeting and internalization in 15 different cancer cell lines including C6 cells, where live cell imaging showed a direct interaction with the plasma membrane. Following internalization in C6 cells, miRNA-221 was efficiently delivered inside the cells and enabled the blockage of miRNA-221 induced cell proliferation resulting in a decrease in cell viability. In C6-xenograft bearing nude mice, AS1411 enabled tumor targeting and miRNA-221 delivery inside tumor cells permitting fluorescent and bioluminescent imaging as well as an effective reduction in tumor volume [70]. AS1411 was grafted to a trimodal imaging probes that consists on magnetic fluorescent rhodamine NPs with a colbalt-ferrite core enabling MRI, and loaded with the radioactive ligand $67 \mathrm{Ga}$-citrate providing high sensitivity regardless to tissue tickness where fluorescence signals can be attenuated. Using confocal microscopy analysis and fluorescent intensity measurements, this study showed that AS1411 improved NPs uptake by C6 cells highly expressing NCL. This formulation was also assessed for tumor targeting in in vivo C6-bearing nude mice. Twenty-four hours after intravenous injection, fluorescent, MRI and scintigraphic analysis showed that AS1411 improved the NPs accumulation in the tumor [71].

AS1411 was also used to target other cancer types such as melanomas, which are difficult to treat because of high rate metastasis and drug resistance. siBRAF (BRAF gene mutation found in $60 \%$ of melanomas) loaded liposomes were conjugated with AS1411 and assessed for melanomas therapy. Confocal laser scanning microscopy and flow cytometry revealed AS1411 potential to target these liposomes to A375 melanomas cells. Quantifying mRNA level by real time PCR (Polymerase Chain Reaction), showed a significant gene silencing and a decrease in BRAF protein expression resulting in cell proliferation suppression. In vivo studies were conducted on nude mice bearing A375 xenograft. Liposomes were injected in tail vain and fluorescence images were taken during 48 hours. A fast and persistent accumulation in the tumor was reported. Real time PCR showed a significant decrease in BRAF mRNA tumors resulting in tumor necrosis assessed by HE (Hematoxylin and Eosin) staining suggesting that AS1411 also efficiently promoted targeting and delivery in-vivo [72].

AS1411 grafted NPs were also investigated for breast cancer therapy, where the major limits of actual treatments, especially chemotherapy and total mastectomy, are side effects that may have psychological impact on women lives, and the high metastatic risk. Hence, NPs and targeted therapies such as NCL are being more and more investigated in breast cancer therapy. AS1411 functionalized human serum albumin NPs were used as drug carrier of PTX. This formulation displayed high cell uptake associated to significant cytotoxicity reported in MCF-7 cancer cell line. Affinity to NCL was demonstrated using flow cytometry on MCF-7 cells. Competition studies with endostatin (ligand of surface NCL) showed that NPs internalization was in part mediated by NCL, and in another part by the same way as non-targeted NPs. Interestingly, significant toxicity was observed in normal cells with $\mathrm{Taxol}^{\circ}$ but not with PTX loaded targeted NPs due to limited uptake and accumulation [73]. In another study, AS1411 was grafted to DOX loaded liposomes. Flow cytometry analysis showed that AS1411 enhanced NPs binding to MCF-7 cells as well as their uptake assessed by confocal microscopy. Enhanced antitumor efficacy was observed in MCF-7 cells and MCF7 xenograft bearing mice, which was attributable to enhanced tumor penetration and cellular internalization by AS1411. Indeed, this study demonstrated in in-vivo, after intratumoral injection and ex vivo, mimicking injection experiments, that AS1411-liposomes were not simply in the interstitial space of the tumor tissues but inside tumor cells [74].

AS1411 grafted to gold nanostars showed an excellent anticancer potential mediated by NCL in a panel of 12 cancer cell lines belonging to four cancer subcategories: carcinoma, sarcoma, melanoma, and glioblastoma. In this work, NCL overexpression in non-nuclear extract of cancer cells was first confirmed using immunoblotting. AS1411-gold nanostars were efficiently uptaken by cancer cells as shown by confocal microscopy analysis and further confirmed by gold content measurement assay using inductively coupled plasma-mass spectrometry (ICP-MS). Interestingly, a similar uptake was observed with fibroblast cells lacking of plasma membrane NCL, suggesting the implication of other endocytosis pathways independent of surfaceNCL as previously reported [55]. Internalized by cancer cells, AS1411gold nanostars succeeded to induce downregulation of Bcl-2 gene expression evaluated by RT-PCR. Interestingly, the effect was only observed in cancer cells but not in fibroblast cells, concordantly with a different uptake mechanism. Bcl-2 downregulation was further associated to apoptosis evaluated by caspase $3 / 7$ activity. The study showed an important reduction in cell viability 72 hours after treatment with AS1411-gold nanostars. In addition, the anticancer effect was drastically potentiated with light-triggered release of AS1411 from gold nanostars owed to a high localized concentration of AS1411detached from the NPs. Finally, AS1411 conjugated to NPs showed higher anticancer potential compared to the free AS1411 exceeding 10 times the concentration, with a $17 \%$ higher average cell death, emphasizing the importance of NPs as a platform to insure stability and high local concentrations of the drug [75]. Using high resolution TEM, the same group reported deformation of the nuclear envelope that correlated in $80 \%$ with the location of AS1411-gold nanoconstruct near the nucleus of HeLa cells. Folding in the nuclear envelope were notably reported with $450 \mathrm{nM}$ of AS1411 loaded NPs, an effect that was absent with the free AS1411 used at the same concentration, emphasizing once again the role of NPs as route for increasing anticancer activity. Interestingly, these foldings correlated with increased apoptotic effect and decreased cell viability. This work also highlights the role of NCL in the trafficking of AS1411 NPs to the intracellular compartment that was only reported in cells with high surface NCL expression emphasizing the advantage of the NCL as a shuttling protein [76].

Finally, many other formulations of AS1411 conjugated NPs were investigated in preclinical studies for cancer therapy and diagnosis such as quantum dots for human glioblastoma [77], vinorelbine loaded PEG-PLGA NPs for breast cancer [78], gold fluorescent for cervical imaging and photodynamic therapy [79]. Very recently, a very elegant experiment have shown that in a mouse model of breast cancer, systemic injection of AS1411-linked gold nanospheres completely inhibited tumour growth with no sign of toxicity [80]. All these studies showed promising preclinical results owed to high tumor targeting facilitated by NCL-AS1411 interaction.

\section{F3 peptide conjugated NPs targeting NCL}

F3 peptide is a 31-amino-acid sequence of the fragment of the high mobility group protein 2 [81]. F3 specifically binds to cell surface NCL and undergoes cell surface to nucleus transport, enabling a payload transport into the target cell nucleus [14]. Indeed, fluorescein-labeled F3 was shown to translocate into the nucleus of MDA-MB-435 cells in vitro and in xenografts studies [81]. F3 promoted targeted delivery of alpha-emitting particles into the nucleus of intraperitoneally growing xenograft tumors, increasing the survival time of the mice [82] 
Compared to other tumor targeting peptide, F3 has the added benefit of binding to angiogenic vessels in addition to tumor cells [14]. Thus, F3 has been used as an effective ligand to mediate NPs targeting to cancer cells and angiogenic blood vessel endothelial cells within the tumor microenvironment for cancer therapy and imaging.

Many formulations of F3 conjugated NPs were investigated for glioma therapy and imaging. F3 conjugation to hydrogel NPs prevented them from being trapped in the acidic lysosomes of $9 \mathrm{~L}$ glioma cells, avoiding their degradation [83]. F3 peptide further conjugated to DOX loaded hydrogel NPs dramatically enhanced their uptake by NCL mediated endocytosis in NCL-overexpressing 9L glioma cell line. Competition studies with free F3 peptide resulting in a blocking of NPs uptake confirmed the NCL targeting selectivity and support the hypothesis of a NCL-mediated endocytosis uptake. In addition, this work demonstrates for the first time the potential of the F3-targeted drug delivery approach to improve the uptake of NPs by drug-resistant cells (human ovarian adenocarcinoma cell line NCI/ADR-RES), that may be associated to the over expression of NCL [84]. In another study, F3 was linked to PTX loaded PEG-PLGA NPs and assessed for glioma therapy in association with the tumor penetrating peptide tLyp-1. Decoration with F3 improved NPs penetration in 3D tumor spheroids of C6 cells as well as in vivo intracranial C6 glioma bearing mice, resulted in a prolonged survival time that was higher in association with tLyp-1 peptide. It is important to note that pharmacokinetic studies conducted on rats after i.v. administration showed a significantly prolonged elimination half-life and decreased clearance rate of the NPs formulation compared to Taxol $^{\circledR}$ [85]. Furthermore, F3 NPs showed their potential in brain tumor imaging. Surgical resection of brain tumors is often compromised by incomplete resection due to lack of adequate visible contrasts or the need for specialized equipment. NPs targeted directly to tumors and loaded with contrast agent or dyes constitute a promising technique to improve intraoperative brain tumor delineation. The first study published to use dye-loaded NPs to cause visible color change in tumor cells used F3 as tumor targeting ligand to glioma cells. F3 grafted to dye loaded polyacrylamide NPs increased cell binding and internalization of NPs into the cytoplasm resulting in a qualitative color change in glioma cells. Therefore, the addition of F3 significantly decreases the dose of NPs necessary to cause color change, emphasizing again the importance of a selective tumor targeting in improving the techniques outcome [86].

F3 NPs were widely investigated in breast cancer studies. Moura et al. studied $\mathrm{pH}$-sensitive liposome functionalized by the F3-peptide in breast cancer models. F3-targeted liposomes were able to bind and internalize human breast cancer cells obtained from patients diagnosed with invasive breast carcinoma. The amount of uptake was only associated with the expression of NCL receptor, regardless of estrogen, progesterone and HER 2 receptor expression, showing the clinical relevance of such strategy for patients resistant to the former blocking receptors therapy regimens such as anti-HER2 (Herceptin $\left.{ }^{\star}\right)$. In vitro, cellular association studies confirmed these results showing the ability of F3 to target liposomes to cells up to 17-fold compared to the non-targeting liposomes. In this study, DOX was encapsulated into the F3-liposomes. This formulation was significantly more effective to induce endothelial (HMEC-1) and breast cancer cell death and to reduce vessel formation with vessel destabilization than nontargeting liposomes, likely owing to the highest intracellular delivery of DOX to targeted cells. F3-targeted liposomes loading DOX were injected in the tail vein of mammary fat pad mice bearing MDA-MB$435 \mathrm{~S}$ tumor. The complex accumulated in the tumor for a time point as short as 4 hours emphasizing the ability of F3 to selectively bind to tumor cells. At 24 hours, tumor accumulation was 33-fold higher than non-targeted counterparts that were preferentially cleared by the spleen and liver, due to lack of targeting specificity. Consistently, preferential accumulation of F3-liposomes in the tumor resulted in a significant reduction in viable rim area and vascular density, induction of cell death and tumor necrosis compared to non-targeting liposomes [87]. In a further study, these F3-liposomes were loaded with a combination of DOX and C6-ceramide, one of the most promising drugs described to inhibit the cancer deregulated pathway of PI3K/ Akt. Acting on different biological and cellular levels of the tumor, the combination targeted to cancer cells by F3, enabled increased cell death against chemotherapy resistant cells MDA-MB-231 as well as sensitive MDA-MB-435S cells, associated to a marked cell and nucleus swelling consistent with necrotic cell death [88]. F3 was also conjugated to carbon nanotubes for near-infrared light therapy of breast cancer. The conjugate was actively internalized in MCF-7 and HAAE-1 endothelial dividing cells resulting in significant cell death further enhanced by near-infrared laser treatment, an effect that was not observed in nondividing confluent endothelial cells [89]. F3 targeted liposomes showed their efficacy to deliver anti-eGFPsiRNA and to down-regulate GFP in MDA-MB-435 cells and angiogenic endothelial cells HMEC- 1 . The success was certainly owed to F3-mediated high cellular association evaluated by flow cytometry and confocal analysis, and that was inhibited at $4^{\circ} \mathrm{C}$, suggesting that a receptor-mediated endocytosis was involved in F3-liposomes uptake [90].

All the reported studies evidently showed the potential of F3 conjugated NPs in cancer therapy and diagnosis. Many other studies evidenced this potential such as cisplatin loaded hydrogel NPs for ovarian cancer therapy [91], hydrogel NPs for tumor-cell targeted photodynamic therapy [92].

\section{N6L conjugated NPs targeting NCL}

Our research team developed several ligands of NCL belonging to a family of molecules named Nucant for NUCleolin ANTagonist. These molecules are composed of a peptidic matrix rich in lysine residues in which several identical pseudotripeptides Lys[CH2N]Pro-Arg are linked [15]. In this context, we generated multivalent pseudopeptide analogues for use in exploring the most efficient size and shape of the matrix and the optimal number of grafted pseudotripeptides. The effects of these compounds on cell growth have been investigated and comparative studies indicated that the optimum inhibitory effect was achieved with N6L which is composed of 6 pseudopeptides. These comparative studies indicated that the specific activity of these molecules is directly linked to the number of pseudotripeptide (unpublished data). N6L, which is currently in phase II clinical trials preparation, interacts to the carboxy-terminal RGG domain of the cell surface NCL inhibiting the binding of NCL ligands including midkine [93], lactoferine [54] and pleiotrophin [94] that constitutes for these molecules a low affinity binding sites.

Like AS1411, N6L has the dual property to specifically target tumor cells and to induce an antitumor effect. Following binding, N6L is internalized and concentrates in the cell nucleolus[15]. This observation has been recently validated using anti-N6L antibodies experiment (unpublished data).

Using several in vitro as well as in vivo models, it has been demonstrated that Nucant induced the apoptosis of tumor cells derived from nearly every type of cancer including $\mathrm{T}$ and $\mathrm{B}$ cell lymphoblastic lymphoma [15], Burkitt lymphoma [15] as well as colorectal [15], breast [95], melanoma [23], glioblastoma and prostate cancer 
(submitted publications). More recently, we obtained data showing that N6L inhibits the growth of tumor derived from pancreatic ductal adenonocarcinoma and decreases the number of metastasis localized in the liver (submitted publication).

Investigations regarding the mechanism of action have indicated that the inhibitory action involves the release of the TIMP-3 from sulfated glycosaminoglycans (GAG) present on the cell surface or within the extracellular matrix, as a high affinity binding of N6L was demonstrated for sulfated GAGs, as well as a displacement of GAGbound TIMP-3 [96] emphasizing that N6L binds to sulfated GAGs. Recently, in order to study the effect of sulfated GAG on the biological activity of N6L, we showed that a stable polyplex nanoparticles with antitumour and anti-metastasis activities were obtained through selfassembly between N6L and sulfated glycosaminoglycans as anionic polymers (unpublished data). In addition, another interesting feature of these results is that a polyplexed structure of N6L improved drastically the efficacy of the antitumor activities of N6L. This feature has been demonstrated in vitro as well as in vivo using several experimental models of tumor growth (submitted publication).

Although the identification of the mechanism of this potentiating effect was not known, these findings are consistent with previous reports related to the biological properties and activities of heparin binding growth factors. Thus, it is now well known that certain sulfated GAG potentiates the activity of several growth factors, such as FGF2 [97], HGF/SC [98], HARP/PTN [99], increasing the affinity and interaction of growth factors to their specific receptor. In addition, it has been shown that sulfated GAGs are involved in the internalisation of these growth factors leading to a perinuclear or nuclear location [100]. The involvement of sulfated GAGs in the internalisation of N6L remains unknown and is currently under investigation. However, according to the data from the literature, it is tempting to speculate that N6L could be translocated to the nucleolus through its interaction with sulphated GAG and as described for LEDGF [101] was facilitated by GAGs. This possibility is also supported by the fact that treatment of cells with a mixture of heparitinases I, II, and III and chondroitinases A, $\mathrm{B}$, and $\mathrm{C}$ decreases the binding of N6L to cell surface and consequently its internalization [96]. The role of GAG in the potentiation of the biological activity of N6L is the focus of our current research.

Within the framework of the European project Multifun. N6L was grafted to iron oxide magnetic NPs for breast cancer detection and treatment. NPs intracellular uptake was successfully increased by N6L in MDA-MB-231 cells and MDA-MB-231 xenograft after intravenous injection. Pull down assay showed that the targeting occurs in part by NCL recognition (unpublished data). N6L also promoted in vitro cell targeting of DOX loaded NPs and improved in vivo tumor volume reduction after intratumoral injection compared to non-targeted DOX loaded NPs [102]. In this study, analysis of the results indicated that N6L functionalization improved cellular uptake and DOX functionalization mediated additional cytotoxicity compared to the non-functionalized nanoparticles. These promising studies encourage the development of other N6L targeted NPs formulations and the investigation in different cancer models.

\section{None conjugated DNA NPs targeting NCL}

DNA NPs are non-viral gene transfer vectors with great potential in vivo. NCL was also shown to serve as a receptor for DNA NPs, not loaded with any of the targeting ligands. In the work of Chen et al., using surface plasmon resonance, it was demonstrated that DNA NPs bind to NCL with an affinity of $25 \mathrm{nM}$. Rhodamine labeled DNA NPs were internalized into HeLa cells. DNA NPs colocalized with NCL on the cell surface, in the cytoplasm and the nucleoli of HeLa cells, showing NCL implication in NPs trafficking from cell surface to nucleus, supported by the fact that NCL shuttles between the different cell compartments. Binding specificity to NCL was confirmed by competition studies with purified NCL and by blocking NCL using siRNA or serum free medium, all resulted in a remarkable decrease in cell transfection (gene activity) [103]. In a further study, the same group reported that DNA NPs uptake was lipid raft-mediated endocytosis and found both NCL and DNA NPs in lipid rafts [104]. Remarkably, NCL is a good target of non-viral gene delivery.

\section{Conclusion}

The present review focuses on NPs binding to NCL as a targeted drug delivery system for cancer therapy and diagnosis. Overexpressed in almost all cancer cell types, NCL is being intensively investigated as a target in cancer therapy. Hence, NCL ligands AS1411, F3 and N6L have attracted attentions because of their specific tumor targeting properties associated to antitumor effects in the case of AS1411 and N6L. Grafted to different formulations of NPs, NCL ligands successfully targeted many different cancer cell lines and xenografts with great selectivity and promoted internalization of the loaded drug and/or contrast agent. These studies were performed compared to controls including nontargeted NPs, normal cell lines, non-dividing confluent endothelial cells, a scrambled F3 sequence, or a non-aptameric DNA sequence. Besides, different techniques and methods were used to prove NPs interaction with NCL.

Nevertheless, these promising studies lack of exploration of the mechanisms behind NPs binding to NCL and internalization, and few studies are investigating to help understand this point that evidently will help to clarify the following trafficking route of the NPs or the NCLNPs complex. Besides, few of these studies reported a translocation of NPs into the nucleus that could remarkably improve the observed results. Finally, despite all the assuring preclinical results, a low number of these NPs has currently entered clinical trials, and it would be useful to push forward the research especially now that AS1411 and N6L have already reached clinical trials.

\section{References}

1. IAfRoC, GLOBOCAN (2012): Estimated Cancer Incidence, Mortality and Prevalence Worldwide in 2012

2. Singla AK, Garg A, Aggarwal D (2002) Paclitaxel and its formulations. Int J Pharm 235: 179-192.

3. Ichikawa Y, Ghanefar M, Bayeva M, Wu R, Khechaduri A, et al. (2014) Cardiotoxicity of doxorubicin is mediated through mitochondrial iron accumulation. J Clin Invest 124: 617-630.

4. Srivatsan A, Chen X (2014) Recent advances in nanoparticle-based nuclea imaging of cancers. Adv Cancer Res 124: 83-129.

5. Drbohlavova J, Chomoucka J, Adam V, Ryvolova M, Eckschlager T, et al. (2013) Nanocarriers for anticancer drugs--new trends in nanomedicine. Curr Drug Metab 14: 547-564.

6. Wilczewska AZ, Niemirowicz K, Markiewicz KH, Car H (2012) Nanoparticles as drug delivery systems. Pharmacol Rep 64: 1020-1037.

7. Lammers T, Aime S, Hennink WE, Storm G, Kiessling Theranosticnanomedicine. Acc Chem Res 44: 1029-1038.

8. Xiao K, Li Y, Lee JS, Gonik AM, Dong T, et al. (2012) "OA02" peptide facilitates the precise targeting of paclitaxel-loaded micellar nanoparticles to ovarian cancer in vivo. Cancer Res 72: 2100-2110.

9. Reddy GR, Bhojani MS, McConville P, Moody J, Moffat BA, et al. (2006) Vascular targeted nanoparticles for imaging and treatment of brain tumors. Clin Cancer Res 12: 6677-6686. 
Citation: Sader M, Courty J, Destouches D (2015) Nanoparticles Functionalized with Ligands of Cell Surface Nucleolin for Cancer Therapy and Diagnosis. J Nanomed Nanotechnol 6: 310. doi:10.4172/2157-7439.1000310

10. Berger CM, Gaume X, Bouvet P (2015) The roles of nucleolin subcellular localization in cancer. Biochimie 113: 78-85

11. Fujiki H, Watanabe T, Suganuma M (2014) Cell-surface nucleolin acts as a central mediator for carcinogenic, anti-carcinogenic, and disease-related ligands. J Cancer Res Clin Oncol 140: 689-699.

12. Koutsioumpa M, Papadimitriou E (2014) Cell surface nucleolin as a target for anti-cancer therapies. Recent Pat Anticancer Drug Discov 9: 137-152.

13. Bates PJ, Laber DA, Miller DM, Thomas SD, Trent JO (2009) Discovery and development of the G-rich oligonucleotide AS1411 as a novel treatment for cancer. Exp Mol Pathol 86: 151-164.

14. Christian S, Pilch J, Akerman ME, Porkka K, Laakkonen P, et al. (2003) Nucleolin expressed at the cell surface is a marker of endothelial cells in angiogenic blood vessels. J Cell Biol 163: 871-878.

15. Destouches D, Page N, Hamma-Kourbali Y, Machi V, Chaloin O, et al. (2011) A simple approach to cancer therapy afforded by multivalent pseudopeptides that target cell-surface nucleoproteins. Cancer Res 71: 3296-3305.

16. Orrick LR, Olson MO, Busch H. (1973) Comparison of nucleolar proteins of normal rat liver and Novikoffhepatoma ascites cells by two-dimensional polyacrylamide gel electrophoresis. Proc Natl Acad Sci USA 70: 1316-1320.

17. Mongelard F, Bouvet $P$ (2007) Nucleolin: a multiFACeTed protein. Trends Cell Biol 17: 80-86

18. Hovanessian AG, Puvion-Dutilleul F, Nisole S, Svab J, Perret E, et al. (2000) The cell-surface-expressed nucleolin is associated with the actin cytoskeleton. Exp Cell Res 261: 312-328.

19. Schwab MS, Dreyer C (1997) Protein phosphorylation sites regulate the function of the bipartite NLS of nucleolin. Eur J Cell Biol 73: 287-297.

20. Semenkovich CF, Ostlund RE Jr, Olson MO, Yang JW (1990) A protein partially expressed on the surface of HepG2 cells that binds lipoproteins specifically is nucleolin. Biochemistry 29: 9708-9713.

21. Sirri V, Pession A, Trerè D, Montanaro L, Derenzini M (1995) Proportionally constant quantitative transmission of nucleolin and protein B23 in cycling cancer cells. Clin Mol Pathol 48: M264-268.

22. Derenzini M, Sirri V, Trerè D, Ochs RL (1995) The quantity of nucleolar proteins nucleolin and protein B23 is related to cell doubling time in human cancer cells. Lab Invest 73: 497-502.

23. El Khoury D, Destouches D, Lengagne R, Krust B, Hamma-Kourbali Y, et al (2010) Targeting surface nucleolin with a multivalent pseudopeptide delays development of spontaneous melanoma in RET transgenic mice. BMC Cancer 10: 325

24. Galzio R, Rosati F, Benedetti E, Cristiano L, Aldi S, et al. (2012) Glycosilated nucleolin as marker for human gliomas. J Cell Biochem 113: 571-579.

25. Goldshmit Y, Trangle SS, Kloog Y, Pinkas-Kramarski R (2014) Interfering with the interaction between ErbB1, nucleolin and Ras as a potential treatment for glioblastoma. Oncotarget 5: 8602-8613.

26. Grinstein E, Wernet P, Snijders PJ, Rösl F, Weinert I, et al. (2002) Nucleolin as activator of human papillomavirus type 18 oncogene transcription in cervical cancer. J Exp Med 196: 1067-1078.

27. Hammoudi A, Song F, Reed KR, Jenkins RE, Meniel VS, et al. (2013) Proteomic profiling of a mouse model of acute intestinal Apc deletion leads to identification of potential novel biomarkers of human colorectal cancer (CRC). Biochemical and biophysical research communications 440: 364-370.

28. Hoja-Åukowicz D, PrzybyÅ,o M, Poche ÄE, Drabik A, Silberring J, et al. (2009) The new face of nucleolin in human melanoma. Cancer Immunol Immunother 58: $1471-1480$

29. Otake Y, Soundararajan S, Sengupta TK, Kio EA, Smith JC, et al. (2007) Overexpression of nucleolin in chronic lymphocytic leukemia cells induces stabilization of bcl2 mRNA. Blood 109: 3069-3075.

30. Pichiorri F, Palmieri D, De Luca L, Consiglio J, You J, et al. (2013) In vivo NCL targeting affects breast cancer aggressiveness through miRNA regulation. $J$ Exp Med 210: 951-968.

31. Qiu W, Zhou F, Zhang Q, Sun X, Shi X, et al. (2013) Overexpression of nucleolin and different expression sites both related to the prognosis of gastric cancer APMIS 121:919-925.
32. Shen N, Yan F, Pang J, Wu LC, Al-Kali A, et al. (2014) A nucleolin-DNMT1 regulatory axis in acute myeloid leukemogenesis. Oncotarget 5: 5494-5509.

33. Soundararajan S, Wang L, Sridharan V, Chen W, Courtenay-Luck N, et al. (2009) Plasma membrane nucleolin is a receptor for the anticancer aptamer AS1411 in MV4-11 leukemia cells. Mol Pharmacol 76: 984-991.

34. Zhao H, Huang Y, Xue C, Chen Y, Hou X, et al. (2013) Prognostic significance of the combined score of endothelial expression of nucleolin and CD31 in surgically resected non-small cell lung cancer. PLoS One 8: e54674.

35. Bywater MJ, Pearson RB, McArthur GA, Hannan RD (2013) Dysregulation of the basal RNA polymerase transcription apparatus in cancer. Nat Rev Cancer 13: 299-314.

36. Shang Y, Kakinuma S, Nishimura M, Kobayashi Y, Nagata K, et al. (2012) Interleukin-9 receptor gene is transcriptionally regulated by nucleolin in T-cell lymphoma cells. MolCarcinog 51: 619-627.

37. Uribe DJ, Guo K, Shin YJ, Sun D (2011) Heterogeneous nuclea ribonucleoprotein $\mathrm{K}$ and nucleolin as transcriptional activators of the vascular endothelial growth factor promoter through interaction with secondary DNA structures. Biochemistry 50: 3796-3806.

38. Zhang J, Tsaprailis G, Bowden GT (2008) Nucleolin stabilizes Bcl-X L messenger RNA in response to UVA irradiation. Cancer Res 68: 1046-1054

39. Ishimaru D, Zuraw L, Ramalingam S, Sengupta TK, Bandyopadhyay S, et al. (2010) Mechanism of regulation of bcl-2 mRNA by nucleolin and A+U-rich element-binding factor 1 (AUF1). J Biol Chem 285: 27182-27191.

40. Chen J, Guo K, Kastan MB (2012) Interactions of nucleolin and ribosomal protein L26 (RPL26) in translational control of human p53 mRNA. J BiolChem 287: $16467-16476$

41. Takagi M, Absalon MJ, McLure KG, Kastan MB (2005) Regulation of p53 translation and induction after DNA damage by ribosomal protein L26 and nucleolin. Cell 123: 49-63.

42. Abdelmohsen K, Tominaga K, Lee EK, Srikantan S, Kang MJ, et al. (2011) Enhanced translation by Nucleolin via G-rich elements in coding and noncoding regions of target mRNAs. Nucleic Acids Res 39: 8513-8530.

43. Kadomatsu K, Muramatsu T (2004) Midkine and pleiotrophin in neura development and cancer. Cancer Lett 204: 127-143.

44. Koutsioumpa M, Polytarchou C, Courty J, Zhang Y, Kieffer N, et al. (2013) Interplay between $\hat{I} \pm v \hat{l}^{2} 3$ integrin and nucleolin regulates human endothelia and glioma cell migration. J Biol Chem 288: 343-354

45. Koutsioumpa M, Drosou G, Mikelis C, Theochari K, Vourtsis D, et al. (2012) Pleiotrophin expression and role in physiological angiogenesis in vivo: potential involvement of nucleolin. Vasc Cell 4: 4

46. Tate A, Isotani S, Bradley MJ, Sikes RA, Davis R, et al. (2006) Met-independent hepatocyte growth factor-mediated regulation of cell adhesion in human prostate cancer cells. BMC Cancer 6: 197.

47. Reyes-Reyes EM, Akiyama SK (2008) Cell-surface nucleolin is a signa transducing P-selectin binding protein for human colon carcinoma cells. Exp Cell Res 314: 2212-2223.

48. Krust B, El Khoury D, Nondier I, Soundaramourty C, Hovanessian AG (2011) Targeting surface nucleolin with multivalent HB-19 and related Nucantpseudopeptides results in distinct inhibitory mechanisms depending on the malignant tumor cell type. BMC Cancer 11: 333.

49. Hovanessian AG, Soundaramourty C, El Khoury D, Nondier I, Svab J, et al. (2010) Surface expressed nucleolin is constantly induced in tumor cells to mediate calcium-dependent ligand internalization. PLoS One 5: e15787.

50. Huang Y, Shi H, Zhou H, Song X, Yuan S, et al. (2006) Theangiogenic function of nucleolin is mediated by vascular endothelial growth factor and nonmuscle myosin. Blood 107: 3564-3571.

51. Hanahan D, Weinberg RA (2011) Hallmarks of cancer: the next generation Cell 144: 646-674.

52. Abdollahi A, Folkman J (2010) Evading tumor evasion: current concepts and perspectives of anti-angiogenic cancer therapy. Drug Resist Updat 13: 16-28.

53. Feron O (2004) Targeting the tumor vascular compartment to improve conventional cancer therapy. Trends Pharmacol Sci 25: 536-542.

54. Legrand D, Vigié K, Said EA, Elass E, Masson M, et al. (2004) Surface nucleolin 
Citation: Sader M, Courty J, Destouches D (2015) Nanoparticles Functionalized with Ligands of Cell Surface Nucleolin for Cancer Therapy and Diagnosis. J Nanomed Nanotechnol 6: 310. doi:10.4172/2157-7439.1000310

Page 8 of 9

participates in both the binding and endocytosis of lactoferrin in target cells. Eur J Biochem 271: 303-317

55. Reyes-Reyes EM, Teng Y, Bates PJ (2010) A new paradigm for aptamer therapeutic AS1411 action: uptake by macropinocytosis and its stimulation by a nucleolin-dependent mechanism. Cancer Res 70: 8617-8629.

56. Borer RA, Lehner CF, Eppenberger HM, Nigg EA (1989) Major nucleolar proteins shuttle between nucleus and cytoplasm. Cell 56: 379-390.

57. Bunka DH, Platonova O, Stockley PG (2010) Development of aptamer therapeutics. CurrOpinPharmacol 10: 557-562.

58. Wu X, Chen J, Wu M, Zhao JX (2015) Aptamers: active targeting ligands for cancer diagnosis and therapy. Theranostics 5: 322-344.

59. Rosenberg JE, Bambury RM, Van Allen EM, Drabkin HA, Lara PN Jr, et al (2014) A phase II trial of AS1411 (a novel nucleolin-targeted DNA aptamer) in metastatic renal cell carcinoma. Invest New Drugs 32: 178-187.

60. Bates PJ, Kahlon JB, Thomas SD, Trent JO, Miller DM (1999) Antiproliferative activity of G-rich oligonucleotides correlates with protein binding. J Biol Chem 274: 26369-26377.

61. Soundararajan S, Chen W, Spicer EK, Courtenay-Luck N, Fernandes DJ (2008) The nucleolin targeting aptamer AS1411 destabilizes Bcl-2 messenger RNA in human breast cancer cells. Cancer Res 68: 2358-2365.

62. Girvan AC, Teng Y, Casson LK, Thomas SD, Jüliger S, et al. (2006) AGRO100 inhibits activation of nuclear factor-kappaB (NF-kappaB) by forming a complex with NF-kappaB essential modulator (NEMO) and nucleolin. Mol Cancer Ther 5: $1790-1799$.

63. Reyes-Reyes EM, Å alipur FR, Shams M, Forsthoefel MK, Bates PJ (2015) Mechanistic studies of anticancer aptamer AS1411 reveal a novel role for nucleolin in regulating Rac1 activation. MolOncol.

64. Maltese WA, Overmeyer JH (2014) Methuosis: nonapoptotic cell death associated with vacuolization of macropinosome and endosome compartments. Am J Pathol 184: 1630-1642.

65. Noaparast Z, Hosseinimehr SJ, Piramoon M, Abedi SM (2015) Tumor targeting with a 99mTc-labeled AS1411 aptamer in prostate tumor cells. J Drug Target.

66. Ma H, Gao Z, Yu P, Shen S, Liu Y, et al. (2014) A dual functional fluorescent probe for glioma imaging mediated by blood-brain barrier penetration and glioma cell targeting. Biochem Biophys Res Commun 449: 44-48.

67. Li J, Zheng H, Bates PJ, Malik T, Li XF, et al. (2014) Aptamer imaging with Cu-64 labeled AS1411: preliminary assessment in lung cancer. Nucl Med Biol 41: 179-185.

68. Ai J, Li T, Li B, Xu Y, Li D, et al. (2012) In situ labeling and imaging of cellular protein via a bi-functional anticancer aptamer and its fluorescent ligand. Anal ChimActa 741: 93-99.

69. Guo J, Gao X, Su L, Xia H, Gu G, et al. (2011) Aptamer-functionalized PEGPLGA nanoparticles for enhanced anti-glioma drug delivery. Biomaterials 32 8010-8020.

70. Kim JK, Choi KJ, Lee M, Jo MH, Kim S (2012) Molecular imaging of a cancertargeting theragnostics probe using a nucleolinaptamer- and microRNA-221 molecular beacon-conjugated nanoparticle. Biomaterials 33: 207-217.

71. Hwang do W, Ko HY, Lee JH, Kang H, Ryu SH, et al. (2010) Anucleolintargeted multimodal nanoparticle imaging probe for tracking cancer cells using an aptamer. J Nucl Med 51: 98-105.

72. Li L, Hou J, Liu X, Guo Y, Wu Y, et al. (2014) Nucleolin-targeting liposomes guided by aptamer AS1411 for the delivery of siRNA for the treatment of malignant melanomas. Biomaterials 35: 3840-3850.

73. Wu J, Song C, Jiang C, Shen X, Qiao Q, et al. (2013) Nucleolin targeting AS1411 modified protein nanoparticle for antitumor drugs delivery. Mol Pharm 10: $3555-3563$

74. Xing H, Tang L, Yang X, Hwang K, Wang W, et al. (2013) Selective delivery of an anticancer drug with aptamer-functionalized liposomes to breast cancer cells in vitro and in vivo. J Mater Chem B Mater Biol Med 1: 5288-5297.

75. Dam DH, Culver KS, Odom TW (2014) Grafting aptamers onto gold nanostars increases in vitro efficacy in a wide range of cancer cell types. Mol Pharm 11: 580-587.

76. Dam DH, Lee JH, Sisco PN, Co DT, Zhang M, et al. (2012) Direct observation of nanoparticle-cancer cell nucleus interactions. ACS Nano 6: 3318-3326.

77. Alibolandi M, Abnous K, Ramezani M, Hosseinkhani H, Hadizadeh F (2014) Synthesis of AS1411-aptamer-conjugated CdTe quantum dots with high fluorescence strength for probe labeling tumor cells. J Fluoresc 24: 1519-1529.

78. Zhou W, Zhou Y, Wu J, Liu Z, Zhao H, et al. (2014) Aptamer-nanoparticle bioconjugates enhance intracellular delivery of vinorelbine to breast cancer cells. J Drug Target 22: 57-66.

79. Ai J, Xu Y, Lou B, Li D, Wang E (2014) Multifunctional AS1411-functionalized fluorescent gold nanoparticles for targeted cancer cell imaging and efficient photodynamic therapy. Talanta 118: 54-60.

80. Malik MT, O'Toole MG, Casson LK (2015) AS1411-conjugated gold nanospheres and their potential for breast cancer therapy. Oncotarget.

81. Porkka K, Laakkonen P, Hoffman JA, Bernasconi M, Ruoslahti E (2002) A fragment of the HMGN2 protein homes to the nuclei of tumor cells and tumor endothelial cells in vivo. ProcNatlAcadSci USA 99: 7444-7449.

82. Drecoll E, Gaertner FC, Miederer M, Blechert B, Vallon M, et al. (2009) Treatment of peritoneal carcinomatosis by targeted delivery of the radiolabeled tumor homing peptide bi-DTPA-[F3]2 into the nucleus of tumor cells. PLoS One 4: e5715.

83. Karamchand L, Kim G, Wang S, Hah HJ, Ray A, et al. (2013) Modulation of hydrogel nanoparticle intracellular trafficking by multivalent surface engineering with tumor targeting peptide. Nanoscale 5: 10327-10344.

84. Qin M, Zong H, Kopelman R (2014) Click conjugation of peptide to hydroge nanoparticles for tumor-targeted drug delivery. Biomacromolecules 15: 3728 3734.

85. Hu Q, Gu G, Liu Z, Jiang M, Kang T, et al. (2013) F3 peptide-functionalized PEG-PLA nanoparticles co-administrated with thyp-1 peptide for anti-glioma drug delivery. Biomaterials 34: 1135-1145

86. Orringer DA, Koo YE, Chen T, Kim G, Hah HJ, et al. (2009) In vitro characterization of a targeted, dye-loaded nanodevice for intraoperative tumor delineation. Neurosurgery 64: 965-971.

87. Moura V, Lacerda M, Figueiredo P, Corvo ML, Cruz ME, et al. (2012) Targeted and intracellular triggered delivery of therapeutics to cancer cells and the tumo microenvironment: impact on the treatment of breast cancer. Breast Cance Res Treat 133: 61-73.

88. Fonseca NA, Gomes-da-Silva LC, Moura V, Simões S, Moreira JN (2014) Simultaneous active intracellular delivery of doxorubicin and C6-ceramide shifts the additive/antagonistic drug interaction of non-encapsulated combination. J Control Release 196: 122-131.

89. Prickett WM, Van Rite BD, Resasco DE, Harrison RG (2011) Vascular targeted single-walled carbon nanotubes for near-infrared light therapy of cancer Nanotechnology 22: 455101.

90. Gomes-da-Silva LC, Santos AO, Bimbo LM, Moura V, Ramalho JS, et al. (2012) Toward a siRNA-containing nanoparticle targeted to breast cancer cells and the tumor microenvironment. Int J Pharm 434: 9-19.

91. Winer I, Wang S, Lee YE, Fan W, Gong Y et al. (2010) F3-targeted cisplatinhydrogel nanoparticles as an effective therapeutic that targets both murine and human ovarian tumor endothelial cells in vivo. Cancer Res 70: 8674-8683.

92. Hah HJ, Kim G, Lee YE, Orringer DA, Sagher O, et al. (2011) Methylene blue conjugated hydrogel nanoparticles and tumor-cell targeted photodynamic therapy. MacromolBiosci 11: 90-99.

93. Said EA, Krust B, Nisole S, Svab J, Briand JP, et al. (2002) The anti-HIV cytokine midkine binds the cell surface-expressed nucleolin as a low affinity receptor. J Biol Chem 277: 37492-37502.

94. Said EA, Courty J, Svab J, Delbé J, Krust B, et al. (2005) Pleiotrophin inhibits HIV infection by binding the cell surface-expressed nucleolin. FEBS $\mathrm{J} 272$ 4646-4659.

95. Destouches D, El Khoury D, Hamma-Kourbali Y, Krust B, Albanese P, et al. (2008) Suppression of tumor growth and angiogenesis by a specific antagonist of the cell-surface expressed nucleolin. PLoS One 3: e2518.

96. Destouches D, Huet E, Sader M, Frechault S, Carpentier G, et al. (2012) Multivalent pseudopeptides targeting cell surface nucleoproteins inhibit cancer cell invasion through tissue inhibitor of metalloproteinases 3 (TIMP-3) release. J BiolChem 287: 43685-43693. 
Citation: Sader M, Courty J, Destouches D (2015) Nanoparticles Functionalized with Ligands of Cell Surface Nucleolin for Cancer Therapy and Diagnosis. J Nanomed Nanotechnol 6: 310. doi:10.4172/2157-7439.1000310

97. Damon DH, Lobb RR, D'Amore PA, Wagner JA (1989) Heparin potentiates the action of acidic fibroblast growth factor by prolonging its biological half-life. $J$ Cell Physiol 138: 221-226.

98. Sakata H, Stahl SJ, Taylor WG, Rosenberg JM, Sakaguchi K, et al. (1997) Heparin binding and oligomerization of hepatocyte growth factor/scatter factor isoforms. Heparan sulfate glycosaminoglycan requirement for Met binding and signaling. J Biol Chem 272: 9457-9463.

99. Vacherot F, Delbé J, Heroult M, Barritault D, Fernig DG, et al. (1999) Glycosaminoglycans differentially bind HARP and modulate its biological activity. J Biol Chem 274: 7741-7747.

100. Bouche G, Gas N, Prats H, Baldin V, Tauber JP, et al. (1987) Basic fibroblast growth factor enters the nucleolus and stimulates the transcription of ribosomal genes in ABAE cells undergoing G0-G1 transition. Proc Natl Acad Sci USA 84: 6770-6774.
101. Fatma N, Singh DP, Shinohara T, Chylack LT Jr (2000) Heparin's roles in stabilizing, potentiating, and transporting LEDGF into the nucleus. Invest Ophthalmol Vis Sci 41: 2648-2657.

102. Kossatz S, Grandke J, Couleaud P, Latorre A (2015) Efficient treatment of breast cancer xenografts with multifunctionalized iron oxide nanoparticles combining magnetic hyperthermia and anti-cancer drug delivery. Breast Cancer Res 17: 66

103. Chen X, Kube DM, Cooper MJ, Davis PB (2008) Cell surface nucleolin serves as receptor for DNA nanoparticles composed of pegylatedpolylysine and DNA. Mol Ther 16: 333-342.

104. Chen X, Shank S, Davis PB, Ziady AG (2011) Nucleolin-mediated cellular trafficking of DNA nanoparticle is lipid raft and microtubule dependent and can be modulated by glucocorticoid. Mol Ther 19: 93-102. 